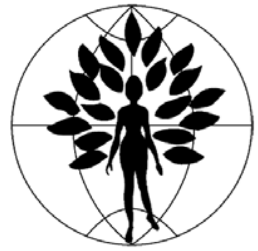

\title{
Modification of the Ingram bicycle seat stool for the treatment of vaginal agenesis and stenosis
}

\author{
Jillian A. Lankford ${ }^{a}$, Hope K. Haefner ${ }^{b, *}$ \\ ${ }^{a}$ Washington University, St Louis, Missouri, USA \\ ${ }^{\mathrm{b}}$ Department of Obstetrics and Gynecology, The University of Michigan Hospitals, Ann Arbor, Michigan, USA
}

\section{KEYWORDS \\ Bicycle seat stool; Neovagina; \\ Vaginal agenesis; \\ Vaginal dilation; \\ Vaginal stenosis}

\begin{abstract}
The use of nonsurgical techniques for the creation of a neovagina for vaginal agenesis and stenosis has been successful for many patients and is currently recommended as first-line therapy. The Ingram bicycle seat stool technique, a nonsurgical approach, has largely replaced the surgical Mclndoe technique as the method of choice for vaginal dilation. It provides an easy and comfortable way for patients to engage in dilation. However, the Ingram bicycle seat stool is not height adjustable. We have created a bicycle seat stool that is height adjustable and will likely provide patients with an improved, more comfortable, and individualized method of dilation.

(C) 2008 International Federation of Gynecology and Obstetrics. Published by Elsevier Ireland Ltd. All rights reserved.
\end{abstract}

\section{Introduction}

Vaginal agenesis is an uncommon condition that affects between 1 in 4000 and 1 in 10000 live born female neonates [1]. Most frequently it is caused by congenital aplasia of the Müllerian ducts and is commonly known as the MayerRokitansky-Kuster-Hauser syndrome. Other less common etiologies of vaginal agenesis include androgen insensitivity syndrome and intersex conditions [2]. Vaginal stenosis, on the other hand, is not a congenital condition and can result from severe infection, ablation and/or radiation due to malignancy, or gender reassignment surgery [3]. Other conditions such as erosive lichen planus can cause complete agglutination of the vagina. Regardless of its cause, a shortened, stenotic, agglu-

\footnotetext{
* Corresponding author. The University of Michigan Hospitals, L 4000 Women's Hospital, 1500 E. Medical Center Drive, Ann Arbor, Michigan 48109, USA. Tel.: +1 734764 8429; fax: +1 7346479727.

E-mail address: haefner@umich.edu (H.K. Haefner).
}

tinated, or absent vagina can be emotionally disturbing for the patient and can result in alterations in self-image and sexual identity [4]. For these reasons, construction or reconstruction of the vagina are often indicated.

Several management options are available for the creation of a neovagina. Numerous surgical options exist, but there is currently no consensus on the best surgical approach. The approach taken is based generally on the experience of the operating surgeon. The most commonly employed surgical approach by gynecologists in the United States is the AbbeMclndoe procedure. This procedure involves placing molds covered with skin grafts (full or split thickness) into a space dissected between the bladder and rectum [5]. Other surgical approaches exist including the use of materials other than skin grafts to cover molds placed into the rectovaginal space, colon or enteric interposition vaginoplasty, vulvovaginoplasty using the labia minora with or without tissue expanders, the Vecchietti and Davydov procedures, as well as laparoscopic modifications of various procedures [6]. Vaginal dilation is required following surgery. 
Despite numerous surgical options for vaginal creation or reconstruction, nonsurgical management options are currently recommended as the first-line approach for the creation of a neovagina [7]. Nonsurgical techniques cause the least morbidity overall [7]. Nonsurgical creation of a neovagina uses a pressure technique in which a vagina is created through the successive use of a series of dilators that gradually increase in diameter and length. The initial technique was described by Frank [8] in 1938. He described the manual use of graduated vaginal dilators placed at the introital dimple for 20 to 30 minutes 3 times daily, for several months. The Frank technique, however, did not gain wide patient acceptance for several reasons, including fatigue of the patient's hands and fingers from holding the dilator in place with constant pressure, the awkwardness of the various positions used (lithotomy, squatting, or Sims position), and the inability of the patient to participate in other activities during the hours that pressure was applied [9].

The problems associated with the Frank method were largely overcome when Ingram introduced a bicycle seat stool model whereby the patient could use their trunk weight in place of manual and digital exertion $[9,10]$. Like the Frank method, the Ingram bicycle seat method of dilation also employs the use of dilators in graduated sizes to create pressure on the vaginal dimple, beginning with a dilator of approximately $1.5 \mathrm{~cm}$ in diameter and $1.5-10 \mathrm{~cm}$ in length. The patient is instructed to use a mirror to examine her genitalia to identify the external structures and the introital dimple. She is then instructed to place the dilator against the introital dimple, hold it in place with a light girdle or spandex underwear, and to sit on the stool, leaning slightly forward, for 30 minutes per day. This method allows the patient to be fully clothed during dilation and also allows her to participate in activities that can be carried out in the sitting position such as studying, reading, computer use, or watching television [10].

The Ingram method of vaginal dilation has been successful in the majority of patients, despite some limitations [7]. The traditional Ingram bicycle seat apparatus for vaginal dilation was a stool with a uniform nonadjustable height. We have created a modified height adjustable bicycle seat stool that will allow comfortable and easy use by patients who require creation of a neovagina and for patients with vaginal strictures. The bicycle seat stool can be adjusted to cover a range of patients' heights.

\section{Description of old and new technologies}

The Ingram bicycle seat stool stands 24 inches tall (just above chair height) and consists of a wooden base made of 2-by-4 inch lumber or a commercial pedestal stool on wheels, a column of pipe, and a bicycle seat of the racing or "male" type $[9,10]$. Our modified bicycle seat stool consists of a 4-pronged aluminum base with rubber feet that provide safety and security for the patient. A column of pipe screws into the aluminum base, thereby making the bicycle seat adjustable for patients of varying heights and leg lengths. A bicycle seat of the racing or "male" type attaches to the adjustable pipe (Fig. 1).

The patient is instructed to use the modified bicycle seat stool in much the same way as the Ingram bicycle seat stool. An initial office visit is required to adjust the angle of the bicycle seat appropriately for proper vaginal dilation.

Recently, a similar nonsurgical treatment of vaginal agenesis was described utilizing a stool with wheels [11]. In this study, the need for a bicycle seat for adequate pressure was questioned, as well as the practical difficulties in manufacturing and handling the bicycle seat stool. Perineal pressure was modulated with postural changes on a regular stool seat instead of a bicycle seat.

\section{Discussion}

There are numerous methods available today to assist in the creation of a neovagina for patients who are affected by vaginal agenesis, vaginal stenosis, or vaginal agglutination. Surgical treatment offers an immediate creation of a neovagina and the Abbe-Mclndoe procedure has led to improved quality of life and sexual satisfaction in some studies [12]. However, these procedures, as well as other surgical procedures, often involve a hospital stay, create a risk for bowel and bladder injury, as well as require the use of vaginal dilation post surgery to maintain the space and prevent stricture [12].

Nonsurgical techniques such as the Ingram method of dilation have been successful in up to $90 \%$ of patients with Müllerian aplasia. They have also been successful when used by patients with vaginal stenosis caused by vaginal contracture following the Abbe-McIndoe procedure, intersex conditions, partial transverse septum, distorted vaginal cuff following hysterectomy, and contracture secondary to colporrhaphy or radiation [13]. The benefits of a nonsurgical bicycle seat stool dilator technique include its simplicity, noninvasiveness, reduced morbidity, and avoidance of hospitalization. However, some complications do exist. Failure of adequate dilation can occur, as well as the potential complication of perforation of

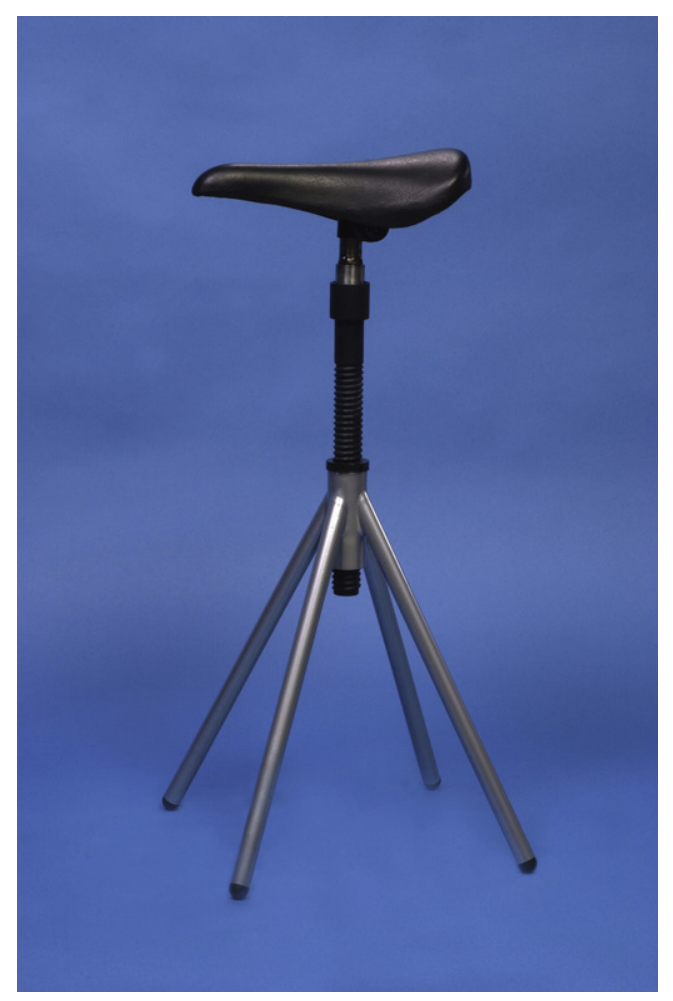

Figure 1 The fully assembled modified Ingram bicycle seat stool. The seat has the ability to change angles to optimize the individual counter-pressure needs of the patient. 
the vagina with possible damage to the bowel or bladder. Rigid dilation has been associated with rectovaginal fistulas in patients who have undergone radiation therapy [14]. Studies have also shown no significant differences between patients treated with dilators versus controls in terms of sexual desire, sexual arousal, and satisfaction with a sexual relationship [15]. Nonsurgical techniques, however, require mature, compliant, and highly motivated individuals who are willing to put in the time and effort required for adequate dilation. Patients can resort to surgical techniques if nonsurgical methods fail.

The modified bicycle seat stool exhibits the same advantages as the Ingram bicycle seat stool with the addition of a height-adjustable seat for patient comfort, as well as a stable aluminum base that provides the patient with safety, security, and ability to maintain dilator traction while seated. The modified bicycle seat stool is also easily disassembled and is lightweight for ease of transport. We have employed its use in patients with vaginal agenesis due to Mayer-RokitanskyKuster-Hauser syndrome as well as in patients who have undergone surgical vaginal reconstruction secondary to lichen planus, requiring an adequate long-term dilation regimen following surgery. Given the recommendation that nonsurgical techniques are the first-line treatment for the creation of a neovagina, the modified bicycle seat stool is an exciting method that will help with the individual needs of patients.

\section{References}

[1] Evans TN, Poland ML, Boving RL. Vaginal malformations. Am J Obstet Gynecol 1981;141(8):910-20.

[2] Tolhurst DE, van der Helm TW. The treatment of vaginal atresia. Surg Gynecol Obstet 1991;172(5):407-14.
[3] Harashina T, Takamatsu A, Inoue Y. Pneumatic vagina dilator. Ann Plast Surg 2004;52(6):621-2.

[4] Foley S, Morely GW. Care and counseling of the patient with vaginal agenesis. Female Patient 1992;17:73-80.

[5] Templeman CL, Lam AM, Hertweck SP. Surgical management of vaginal agenesis. Obstet Gynecol Surv 1999;54(9):583-91.

[6] Templeman C, Hertweck SP. Vaginal agenesis: an opinion on the surgical management. J Pediatr Adolesc Gynecol 2000;13(3): $143-4$.

[7] ACOG Committee on Adolescent Health Care. ACOG Committee Opinion No. 355: Vaginal agenesis: diagnosis, management, and routine care. Obstet Gynecol 2006;108(6):1605-9.

[8] Frank RT. The formation of an artificial vagina without an operation. Am J Obstet Gynecol 1938;35:1053-5.

[9] Williams JK, Lake M, Ingram JM. The bicycle seat stool in the treatment of vaginal agenesis and stenosis. J Obstet Gynecol Neonatal Nurs 1985;14(2):147-50.

[10] Ingram JM. The bicycle seat stool in the treatment of vaginal agenesis and stenosis: a preliminary report. Am J Obstet Gynecol 1981;140(8):867-73.

[11] Lee $\mathrm{MH}$. Non-surgical treatment of vaginal agenesis using a simplified version of Ingram's method. Yonsei Med J 2006;47(6): 892-5.

[12] Klingele CJ, Gebhart JB, Croak AJ, DiMarco CS, Lesnick TG, Lee RA. McIndoe procedure for vaginal agenesis: long-term outcome and effect on quality of life. Am J Obstet Gynecol 2003;189(6):1569-72 discussion 1572-1573.

[13] Roberts CP, Haber MJ, Rock JA. Vaginal creation for mullerian agenesis. Am J Obstet Gynecol 2001;185(6):1349-52 discussion 1352-1353.

[14] Hoffman MS, Wakeley KE, Cardosi RJ. Risks of rigid dilation for a radiated vaginal cuff: two related rectovaginal fistulas. Obstet Gynecol 2003;101:1125-6.

[15] Nadarajah S, Quek J, Rose GL, Edmonds DK. Sexual function in women treated with dilators for vaginal agenesis. J Pediatr Adolesc Gynecol 2005;18(1):39-42. 